\title{
Research Paper: Scorpion Stings in Tehran Province, Iran: A Seven-year Hospital-based Study
}

\author{
Mitra Rahimi ${ }^{1}$ (D), Shahin Shadnia1 ${ }^{1}$, Reza Mirzaei Nasab ${ }^{1}$ (D), Kambiz Soltaninejad²* \\ 1. Department of Clinical Toxicology, School of Medicine, Toxicological Research Center, Excellence Center of Clinical Toxicology, Loghman Hakim \\ Hospital, Shahid Beheshti University of Medical Sciences, Tehran, Iran. \\ 2. Department of Forensic Toxicology, Legal Medicine Research Center, Legal Medicine Organization, Tehran, Iran.
}

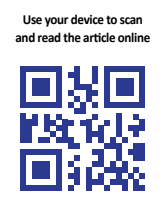

dtation: Rahimi M, Shadnia Sh, Mirzaei Nasab R, Soltaninejad K. Scorpion Stings in Tehran Province, Iran: A Sevenyear Hospital-based Study. International Journal of Medical Toxicology and Forensic Medicine. 2020; 10(4):30274. https://doi. org/10.32598/ijmtfm.v10i4.30274

https://doi.org/10.32598/ijmtfm.v10i4.30274

Article info:

Received: 3 May 2020

First Revision: 25 May 2020

Accepted: 7 Jul 2020

Published: 12 Dec 2021

\section{Keywords:}

Scorpions, Scorpion stings, Antivenin, Iran

\section{ABSTRACT}

Background: Scorpion sting is an important public health problem in Iran. This study aimed to describe the demographics, clinical and laboratory findings of the scorpion stings victims in Tehran Province.

Methods: This cross-sectional retrospective study evaluated demographic characteristics, clinical presentations, laboratory findings, treatments, and disposition of scorpion sting patients in Tehran Province, Iran, during 7 years (2012-2018).

Results: A total of 199 cases of scorpion sting cases with a Mean \pm SD age of $30 \pm 18.7$ years were studied. The majority of patients were males $(62.8 \%)$. The most affected age group was 20-30 years $(31.1 \%)$. The majority of victims $(45.7 \%)$ were residents in the southern districts of Tehran. The yellow scorpions (33.2\%) were responsible for a larger number of envenomations. Most of the stings have been occurred in summer (68.8\%). Also, the most affected limbs were hands and legs (94.4\%). Most patients (58.8\%) had localized manifestations, and the common systemic clinical presentations were hypotension (41.7\%) and hypertension (17.1\%). Also, the common laboratory findings increased blood urea nitrogen (48.7\%), hyperglycemia (20.1\%), and anemia (15.1\%). The polyvalent anti-scorpion serum had been administered for $33.2 \%$ of victims. All patients had been recovered and were discharged in good condition without any complications. There was no fatality report.

Conclusion: The demographic characteristics of scorpion sting in Tehran Province are similar to those previously reported from other Iran provinces. It seems that the incidence and clinical severity of scorpionism in Tehran are lower than those compared to other tropical and subtropical zones in Iran. 


\section{Introduction}

corpion sting is a public health challenge and one of the most critical health problems in tropical and subtropical countries, including Iran [1-3]. Globally, of more than 1500 scorpion species, only 52 species are harmful from the medical and public health perspective $[2,4]$. Annually, more than 1200000 scorpion envenomations with approximately 3200 fatalities are reported in the world $[5,6]$. The most poisonous scorpion species have been reported from the north and south Africa, southern Latin America, Mexico, east of the Andes, the Middle East, and south India [5, 6].

In Iran, 52 species of scorpions, including 11 poisonous species, are recognized [2]. Approximately 4000050000 cases of scorpionism (scorpion envenoming syndrome) have been reported, with nearly 20 fatalities in Iran, annually $[5,7]$. The majority of the scorpion stings victims are reported from the central, western, southwestern, and southern provinces of Iran $[4,5,8]$.

The major poisonous species of scorpions in Iran belong to the Buthidae, Scorpionidae, and Hemiscorpiidae families. Generally, Androctonus crassicauda, Mesobuthus eupeus, Odontobuthus doriae (Buthidae), and Hemiscorpius lepturus (Hemiscorpiidae) are considered medically important scorpions. H. lepturus and A. crassicauda are among the most dangerous scorpion species with fatality during envenomation [8].

Because of diverse clinical presentations of scorpion envenomation, ranging from mild local to severe and fatal outcomes, scorpion sting can be a challenge in emergency medicine practice [8]. Previous studies in Iran showed the various clinical presentations in the scorpion victims. These include local and systemic symptoms. The local symptoms comprise mild local pain, pruritus, erythema, inflammation, blisters, ecchymosis, numbness of limbs, cellulitis, skin necrosis at the site of stings. The systemic symptoms include central and peripheral nervous system (drowsiness, fatigue, irritability, restlessness, decreased tendon reflexes, miosis, convulsion, decreased level of consciousness), cardiovascular (tachycardia, hypotension, hypertension, ECG changes), gastrointestinal system (nausea, vomiting, diarrhea, dry mouth, severe abdominal cramps, melena, elevated liver enzymes), pulmonary (tachypnea, wheezing, acute pulmonary edema), renal (e.g. urination, hematuria, hemoglobinuria, myoglobinuria, proteinuria, acute renal failure), hyperthermia, hypothermia, pallor, sweating, muscle spasms, and cyanosis $[4,7,8]$. Geographical and climatic conditions, scorpion species and behavior, victim characteristics (e.g. age, sex) are responsible for clinical presentations in scorpionism $[2,8]$.

Epidemiological and clinical evaluations in scorpion sting cases are valuable and may improve the therapeutic plans and effective interventions. Variation in the severity of scorpion envenomation should be considered in the training of health care providers to accurately determine the characteristics of endemic scorpion in an area to select a proper therapeutic plan [7]. Despite many studies conducted on scorpionism in some at-risk provinces of Iran, the epidemiological features along with clinical and paraclinical findings in scorpion sting victims in Tehran Province is not clear. Because of the inadequate information in this regard, the current study aimed to determine the demographics, clinical manifestations, and laboratory findings of scorpion sting cases in Tehran Province for 7 years.

\section{Materials and Methods}

\section{Study design and setting}

In this cross-sectional retrospective study, we evaluated the baseline demographics, clinical features, paraclinical findings, clinical management, and outcomes of scorpion sting cases who referred to Loghman Hakim Hospital Poison Center (LHHPC) (as an academic referral hospital) in Tehran Province, Iran, during 2012-2018. The Ethics Committee of Shahid Beheshti University of Medical Sciences approved our study protocol.

\section{Study participants}

We reviewed the records of all scorpion sting patients referring to the LHHPC (both outpatients and inpatients) with available data on the Hospital Information System (HIS). Patients with missing data were excluded from the study.

\section{Data collection}

A database, including demographic information (sex, age, and residential location), scorpion color, sting site, vital signs, clinical manifestations, received treatments in prehospital and in-hospital settings (antivenom, antibiotics, corticosteroids, etc.), laboratory findings, and outcomes of patients were extracted and recorded by a trained physician.

\section{Statistical analysis}

Statistical analyses were performed with SPSS version 16. The descriptive findings were presented as frequency and percentage or Mean \pm SD. Also, we used the Mann- 
Whitney U test, Fisher exact test, Pearson Chi-square test, and Student $t$ test to analyze data. A P-value of less than 0.05 was considered statistically significant.

\section{Results}

The total number of scorpion sting cases during the study period was 199 . The 7-year incidence was esti- mated at 1.53 per 100000 . The figure was calculated from the scorpion sting frequency (199 cases) and the population of Tehran Province (about 13000000).

The gender distribution showed that males were the main affected due to stings compared to females (male: female ratio was 1.69:1). The Mean \pm SD age of the pa-

Table 1. Baseline characteristics of scorpion sting cases

\begin{tabular}{|c|c|c|}
\hline \multicolumn{2}{|c|}{ Variables } & No. (\%) \\
\hline \multirow{3}{*}{ Sex } & Male & $125(62.8)$ \\
\hline & & \\
\hline & Female & $74(37.2)$ \\
\hline \multirow{7}{*}{ Age (y) } & $0-10$ & 35 (17.6) \\
\hline & $10-20$ & $25(12.6)$ \\
\hline & $20-30$ & $62(31.1)$ \\
\hline & & \\
\hline & $30-40$ & $32(16.1)$ \\
\hline & $40-50$ & $25(12.5)$ \\
\hline & $50-70$ & $20(10.1)$ \\
\hline \multirow{8}{*}{$\begin{array}{l}\text { Time from envenomation to admission to } \\
\text { hospital (h) }\end{array}$} & $0-3$ & $131(65.8)$ \\
\hline & $4-6$ & $22(11)$ \\
\hline & $7-9$ & $14(7.1)$ \\
\hline & $10-12$ & $12(6.1)$ \\
\hline & $13-18$ & $1(0.5)$ \\
\hline & $19-24$ & $7(3.5)$ \\
\hline & $25-48$ & $7(3.5)$ \\
\hline & $>48$ & $5(2.5)$ \\
\hline \multirow{6}{*}{ Residential location } & North & $17(8.6)$ \\
\hline & South & 91 (45.7) \\
\hline & Tehran & $26(13)$ \\
\hline & West & $8(4)$ \\
\hline & Center & $20(10)$ \\
\hline & Outside of Tehran & $37(18.7)$ \\
\hline \multirow{3}{*}{ Prehospital management } & None & $144(72.4)$ \\
\hline & Self-therapy (Incision, Suction) & $9(4.5)$ \\
\hline & $\begin{array}{l}\text { Treatment in another clinic before admis- } \\
\text { sion to hospital }\end{array}$ & $46(23.1)$ \\
\hline
\end{tabular}


Table 2. Clinical presentations of scorpion sting victims (N=199)

\begin{tabular}{|c|c|c|}
\hline Clinical Findings & & No. (\%) \\
\hline \multirow{5}{*}{ Local presentations } & Burning sensation & 79 ( 39.7) \\
\hline & Pain & 67 (33.7) \\
\hline & & \\
\hline & Erythema & $66(33.2)$ \\
\hline & Swelling & $66(33.2)$ \\
\hline \multirow[b]{10}{*}{ Systemic presentations } & Sinus Tachycardia & $9(4.5)$ \\
\hline & Sinus Bradycardia & $1(0.5)$ \\
\hline & Hypertension & $34(17)$ \\
\hline & & \\
\hline & Hypotension & $83(41.7)$ \\
\hline & Tachypnea & $15(7.5)$ \\
\hline & Bradypnea & $1(0.5)$ \\
\hline & Paresthesia & 23 (11.5) \\
\hline & Agitation & $7(3.5)$ \\
\hline & Diplopia & $6(3)$ \\
\hline \multirow[t]{4}{*}{ Neurologic } & Vertigo & $6(3)$ \\
\hline & Headache & $8(4)$ \\
\hline & Lethargy & $1(0.5)$ \\
\hline & Carpopedal spasms & $3(1.5)$ \\
\hline \multirow[b]{3}{*}{ Heat regulation system } & Nausea and vomiting & $2(1)$ \\
\hline & Hyperthermia & $4(2)$ \\
\hline & Hypothermia & $1(0.5)$ \\
\hline \multirow{5}{*}{ Sting Severity Grading } & Grade I & $139(70)$ \\
\hline & Grade II & $44(22)$ \\
\hline & & \\
\hline & Grade III & $15(7.5)$ \\
\hline & Grade IV & $1(0.5)$ \\
\hline
\end{tabular}

Sinus tachycardia: defined as the sinus rhythm with a rate greater than 100 beats per minute;

International Journal of Medical Toxicology \& Forensic Medicine Sinus bradycardia: defined as a sinus rhythm with a rate below 60 beats per minute;

Hypertension: defines as elevated blood pressure, with a systolic pressure (SBP) between 120 and $129 \mathrm{~mm} \mathrm{Hg}$ and diastolic pressure (DBP) less than $80 \mathrm{~mm} \mathrm{Hg}$, and stage 1 hypertension, with an SBP of 130 to $139 \mathrm{~mm} \mathrm{Hg}$ or a DBP of 80 to $89 \mathrm{~mm} \mathrm{Hg}$; Hypotension: defined as blood pressure lower than $90 \mathrm{~mm} \mathrm{Hg}$ systolic or $60 \mathrm{~mm} \mathrm{Hg}$ diastolic;

Tachypnea: defined as a respiratory rate greater than 20 breaths per minute;

Bradypnea: defined as a respiration rate below 12 breaths per minute;

Hyperthermia: defined as a core body temperature greater than $37.5^{\circ} \mathrm{C}$;

Hypothermia: defined as a core body temperature below $35^{\circ} \mathrm{C}$. 
Table 3. Common laboratory findings in scorpion stung patients ( $\mathrm{N}=199)$

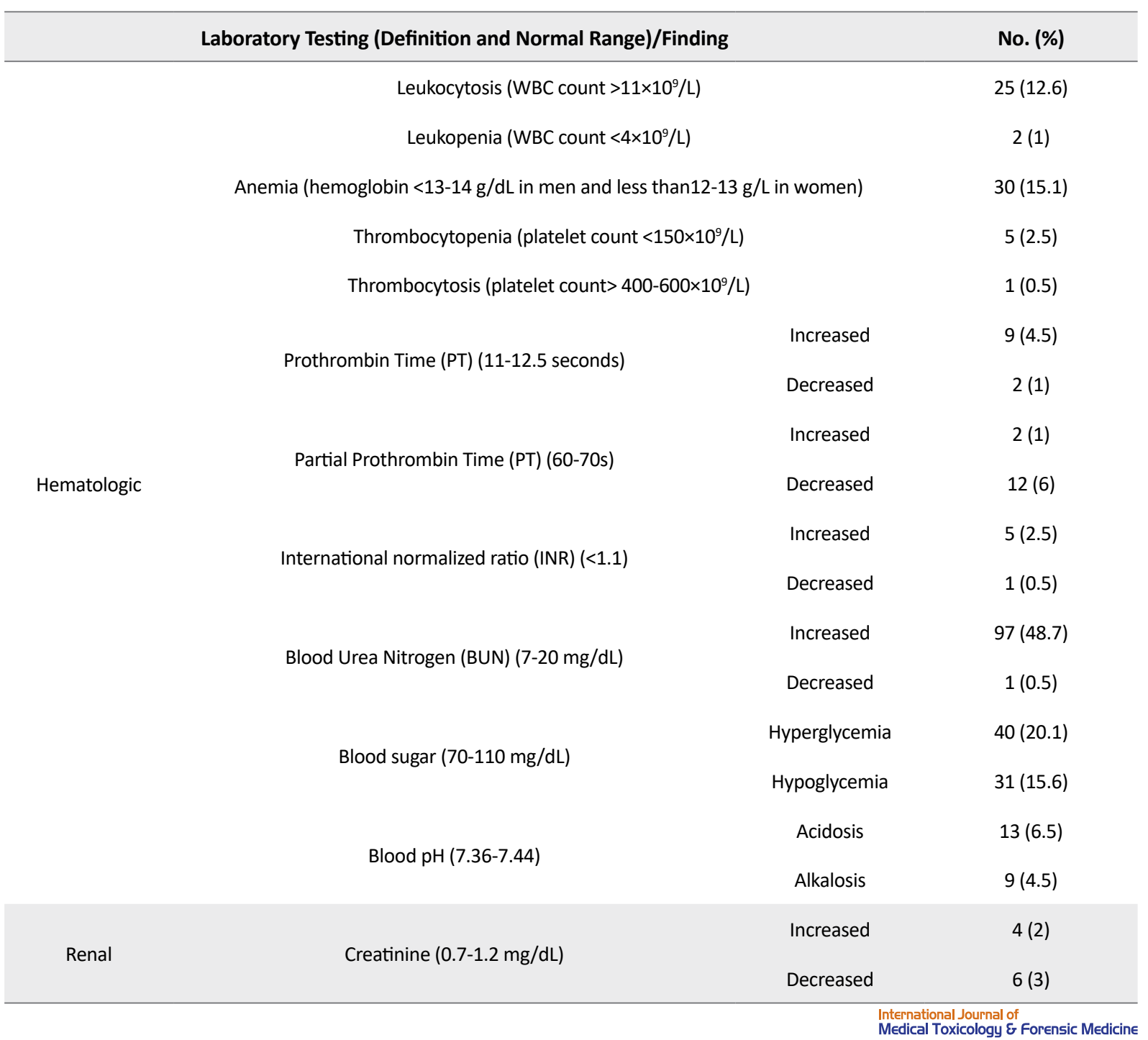

tients was 30 (18.7) years, and the most affected age group was 20-30 years (Table 1).

Most patients had been referred to the hospital within 3 hours after envenomation. Most of the victims were residents in the southern districts of Tehran. Most cases were referred from Tehran Province, and only $18.7 \%$ of patients were from other provinces (Table 1).

The yellow scorpions $(n=66,33.2 \%)$ were responsible for a larger number of envnomations than the black scorpions ( $n=41,20.6 \%)$ and in the rest of situations, color of scorpion had not been mentioned. Most scorpion sting cases occurred in summer $(n=137,68.8 \%)$, followed by spring $(n=44,22.1 \%)$, autumn $(n=14,7.1 \%)$ and winter $(n=4,2 \%)$.

Also, the most affected limbs were hands and legs (94.4\%). The most prevalent sites of sting were the lower extremities ( $n=94,47.2 \%)$, followed by upper extremities $(n=77,38.7 \%)$, trunk $(n=5,2.5 \%)$, and head and neck $(\mathrm{n}=5,2.5 \%)$. Also, in 18 cases $(9 \%)$, the sting site was not determined.

Table 2 summarizes the clinical presentations of the patients when admitted to the hospital. The majority of the patients had localized manifestations, including burning sensation, mild to severe pain, and local erythema and swelling. The typical clinical signs and symptoms were hypotension and hypertension (Table 2).

Based on the previously defined scorpion sting severity grading [8], most of the patients were classified into grade I (Table 2). Hypertension $(n=4,2 \%)$, diabetes mellitus $(n=1,0.5 \%)$, hypothyroidism $(n=1,0.5 \%)$ and asthma $(n=1,0.5 \%)$ were observed as past medical history in the patients. But there is no significant difference 
Table 4. Comparison of clinical manifestations and paraclinical findings of scorpion stings with respect to the scorpion color

\begin{tabular}{|c|c|c|c|}
\hline \multirow{3}{*}{ Variables } & & & \multirow{3}{*}{$\mathbf{P}$} \\
\hline & \multicolumn{2}{|c|}{ Color of Scorpion } & \\
\hline & Yellow $(n=66)$ & Black $(n=41)$ & \\
\hline Local pain & $42(63.6)$ & $27(65.8)$ & 0.84 \\
\hline Burning sensation & $24(36.4)$ & $20(48.8)$ & 0.23 \\
\hline Swelling & $22(33.3)$ & $20(48.8)$ & 0.08 \\
\hline Sinus tachycardia & $28(42.4)$ & $9(22)$ & 0.02 \\
\hline Hypertension & $9(13.6)$ & $6(14.6)$ & 0.86 \\
\hline Hypotension & $28(42.4)$ & $21(51.2)$ & 0.90 \\
\hline Hyperthermia & $2(3)$ & $2(4.9)$ & 0.28 \\
\hline Paresthesia & $6(9.1)$ & $9(22)$ & 0.03 \\
\hline Agitation & $2(3)$ & $3(7.3)$ & 0.45 \\
\hline Diplopia & $0(0)$ & $3(7.3)$ & 0.03 \\
\hline Vertigo & $4(6.1)$ & $0(0)$ & 0.01 \\
\hline Headache & $1(1.5)$ & $1(2.4)$ & 0.54 \\
\hline Lethargy & $0(0)$ & $1(2.4)$ & 0.85 \\
\hline Carpopedal spasms & $1(1.5)$ & $2(4.9)$ & 0.75 \\
\hline Nausea and vomiting & $1(1.5)$ & $1(2.4)$ & 0.54 \\
\hline Grade I & $53(80.3)$ & $22(53.6)$ & 0.01 \\
\hline Grade II & $10(15.1)$ & $14(34.1)$ & 0.01 \\
\hline Grade III & $2(3)$ & $4(9.7)$ & 0.01 \\
\hline Grade IV & $0(0)$ & $1(2.4)$ & 0.84 \\
\hline
\end{tabular}

in clinical presentations according to this history in the victims $(\mathrm{P}>0.05)$.

The common laboratory findings were increased blood urea nitrogen (BUN) (48.7\%), hyperglycemia (20.1\%), anemia (15.1\%), and leukocytosis (12.6\%) (Table 3). No prehospital first aid measures had been taken in 144 cases (72.4\%); among first aid measures taken, incision and suction of sting site had been done the most (Table 1).

Polyvalent anti-scorpion serum (Razi vaccine and serum research institute, Karaj, Iran) had been administered for 66 victims $(33.2 \%)$. The majority of victims $(n=42,63.6 \%)$ were given only one vial of antivenom, and the rest of them were given more than one vial
(29.2\% of cases were given 2 vials, $4.6 \%$ were given 3 vials, and $1.5 \%$ [one case] was given 6 vials).

The other medications administered included systemic corticosteroids $(41.7 \%)$, antihistamines $(41.2 \%)$, antiemetics $(43.2 \%)$, analgesics $(35.7 \%)$, tetanus antitoxin $(21.6 \%)$, sedatives $(29.1 \%)$, local anesthetics $(4.5 \%)$, antibiotics (12.6\%).

The clinical manifestations of scorpion stings, according to the scorpions' color, are presented in Table 4. Sinus tachycardia was more prevalent in stings with yellow than black scorpions. Moreover, paresthesia and diplopia were common in sting with black scorpion than yellow scorpion (Table 4). Also, there were no significant differences between the two groups regarding age $(\mathrm{P}=0.17)$, 
location $(\mathrm{P}=0.41)$, time of hospital admission $(\mathrm{P}=0.23)$, sting site $(\mathrm{P}=0.90)$, and laboratory findings. However, there was a significant difference between the two groups regarding the grading of the stings. Black scorpion stings caused more severe symptoms in comparison with yellow scorpion envenomations $(\mathrm{P}=0.01)$. Administered scorpion antivenin to the patients created significant differences between two groups $(\mathrm{P}=0.02)$.

All patients were recovered and discharged in good condition without any complications. There was no fatality.

\section{Discussion}

The main findings of this study showed that the majority of the scorpion sting victims in Tehran during this period were males. They were mostly 20-30 years old, mainly residents in the southern region of the province with mild to moderate local and systemic clinical presentations and laboratory findings (grade I and II) without any case fatality.

The overall incidence of scorpion stings in Tehran Province during the study period was low compared to previous studies in Iran $[5,8,9]$. For example, Khatony et al. showed a high incidence of scorpion stings with 334.37 per 100000 and 339.07 per 100000 population in tropical areas of Kermanshah Province (the cities of Ghasre-Shirin, Gilan-e Gharb and Sarpol-e Zahab) in 2008 and 2009, respectively [5]. A previous study showed that the 5-year prevalence of scorpion sting was 9.1 per 1000 inhabitants in Andimeshk District (Khuzestan Province, Iran) during 2012-2016 [9]. This difference should be related to geographic distribution of poisonous scorpion species and climatic conditions between two regions of the country $[2,5,8,14]$. Scorpion's species, age, and size are the important factors in severity of scorpion envenomation [14].

In our study, the scorpion stings had occurred mainly in males with the age group of 20-30 years. This finding is in concordance with the results of most previous studies in Iran and other countries [5, 8-13]. This result could be due to the job and social activity of men in comparison with women [10].

In the current study, the arrival time to the hospital after scorpion sting was mostly within the first three hours, and this finding is similar to the previous reports $[7,8]$. In this study, lower and upper extremities were the commonest sting sites than other parts of the body. This finding is similar to previously reported scorpion causalities in Iran [7-9]. It could be related to the fact that people of the studied regions, especially those residents in the south of Tehran Province, wear unsuitable footwear and employed with handiwork jobs in scorpion living areas due to their socio-economic status [9].

In our study, the highest incidences of scorpion stings were recorded in the summer and spring. The finding is in concordance with other previous studies in several areas of Iran and in neighboring countries that indicated a high incidence of scorpion stings in the summer [4, 8, 10, 15].

Yellow scorpions were responsible for most of the stings in our study; however, the scorpions' color remained unknown in many cases. Other studies from Iran have reported similar results $[7,8]$. It is challenging to identify scorpion species just based on their color. However, previous studies report that the main poisonous yellow scorpions, including Mesobuthus eupeus and Odontobuthus doriae, belong to the Buthidae family are mainly distributed in Tehran. Also, Androctonus crassicauda as a black scorpion has been reported from Tehran [4].

In the present study, local clinical presentations, including burning sensation, pain, and, erythema were the most common signs and symptoms in the patients. This result is similar to previous reports $[7,8,15]$. These findings should be related to the biological activity of scorpion venoms. The toxicity of scorpions is related to the compositions of their venoms as an essential source of bioactive components, including toxins and other small peptides. For example, a previous study showed that the venom of Mesobuthus eupeus as the most abundant species in Iran, including toxins, cell proteins, unknown peptides, and various toxin families with effect on ion channels [16].

Tachycardia and hypertension were common systemic clinical presentations in our study. These observations were in line with the previous studies, which showed that the venom of scorpion stimulated the sympathetic system and increased blood pressure and heart rate [7, 17]. The scorpion venom stimulates catecholamine release and overstimulates the sympathetic system. Also, we observed hypotension as a common cardiovascular presentation in our patients. This symptom may be due to disturbances in the tone of sympathetic and parasympathetic systems during scorpion envenomation [17].

In our study, severe complications such as seizure, dysrhythmia, renal failure, or fatality were not been observed. These findings have been mentioned in previous studies [7, 9, 13, 15]. For example, Cesaretli et al., in a study based on data recorded in the national poison information center of Turkey, showed that the scorpion sting 
victims at the hospital admission had localized signs, including pain, numbness, hyperemia and edema and mild to moderate systemic effects such as hyperthermia, nausea and vomiting, tachycardia, shivering and lethargy. Still, no lethality was reported [15]. In another study, localized pain, redness, and swelling were the most frequent complaints in most scorpion sting patients. Besides, all patients were found to be grade I envenomation [13].

In our patients, laboratory findings, including increased BUN, hyperglycemia, anemia, leukocytosis, acidosis, partial thromboplastin time (PTT), PT, and thrombocytopenia, were notable. There are scant data on the alterations of clinical laboratory parameters in scorpion envenomation victims in Iran. Previous studies have focused on Hemiscorpius lepturus and Androctonus crassicauda envenomations. Leukocytosis, anemia, lymphopenia, and coagulopathies have been reported in victims of these scorpion stings $[2,18]$. Hyperglycemia is another laboratory finding in our study. Previous studies suggested that the presence of hyperglycemia is an indicator of the severity of scorpion stings and was associated with the severity of clinical manifestations, including respiratory failure, pulmonary edema, hemodynamic instability, neurological failure, and multisystem organ failure, which requires intensive care unit (ICU) admission [19]. Scorpionism induces an autonomic disturbance by releasing catecholamines and increased angiotensin II, glucagon, cortisol, and thyroid hormones. Suppression of insulin levels or induction of insulin resistance as a consequence of hyperinsulinemia and increased circulating free fatty acid levels have been shown as mechanisms of hyperglycemia in scorpionism [19, 20].

Bouaziz et al. found that the laboratory indicators such as leukocytosis of more than 25000 cells $/ \mathrm{mm} 3$ and blood urea level above $8 \mathrm{mmol} / \mathrm{L}$ were correlated with a poor outcome in scorpion stings [21].

The results of our study showed that the clinical severity in black scorpion stings victims was worse in comparison with yellow scorpion stings. This finding was supported with previous similar results [8]. Clinical management of scorpion sting is based on supportive and symptomatic measures, including fluid and electrolyte therapy, administration of antibiotics, analgesics, tetanus immunization, and other medications with specific anti-scorpion serum as antivenom therapy [7]. However, the use of antivenom has been challenged because it could induce allergic reactions. Nevertheless, antivenom therapy is commonly considered as the most effective modalities in scorpion sting [22].

In Iran, scorpion antivenom is a polyvalent immune serum derived from the purified plasma of immunized horses against venoms of the 6 medically important scorpion species in Iran: O. doriae, M. eupeus, A. crassicauda, Buthotus saulcyi, Buthotus schach, and H. lepturus [8]. In sting cases presenting without systemic signs and symptoms, administration of one vial of scorpion antivenin is advised. The decision to administer more antivenom should be made according to the clinical presentations. However, in patients with severe systemic or general symptoms, 3-6 intravenous antivenom should be administered [8, 23].

In our study, only $32 \%$ of the patients received antivenom. The majority of cases were treated with supportive and symptomatic measures because of their mild and moderate clinical presentations and severity of scorpionism in the patients. This result is different from previous studies in Iran in which antivenom was used in the majority of scorpion stings $[5,7,8,12]$. Also, the different number of the received scorpion antivenin vials according to scorpion color should be considered due to various clinical severity scoring in these groups [8].

Since the severity of most scorpion stings was grade I, we suggest that scorpion sting cases can be treated in the outpatient departments because, like other geographical regions in Iran, H. lepturus and A. crassicauda are scarce in Tehran Province. In the present study, we did not have any fatality due to scorpion stings. This finding is in line with previous studies that reported no lethality $[5,7-9,15,24]$.

There are some limitations in our study. For example, scorpion sting cases treated in emergency departments of other hospitals or referred to clinical centers for treatment due to the mild symptoms were not included in this study. Aside from that, scorpion stings are more prevalent in Tehran Province. However, like any other retrospective studies, some data were missing in some of the patients' medical records.

\section{Conclusion}

Scorpion sting is still a medical problem in Iran and planning educational, public health, and medical strategies for its prevention and management is necessary. Although the majority of scorpion sting cases that occurred in Tehran Province were mild to moderate, continuous educations for medical care providers, especially for the staff of Emergency Departments, is necessary.

\section{Ethical Considerations}




\section{Compliance with ethical guidelines}

This study ethically was approval by the Medical Ethics Committee of the School of Medicine, Shahid Beheshti University of Medical Sciences (SBUMS) (Ethics Code: IR.SBMU.MSP.REC.1395. 464).

\section{Funding}

This research did not receive any specific grant from funding agencies in the public, commercial, or non-profit sectors.

\section{Author's contributions}

Conceptualization and supervision: Mitra Rahimi and Shahin Shadnia; Methodology: Mitra Rahimi and Reza Mirzaei Nasab; Data collection: Reza Mirzaei Nasab; Data analysis: Kambiz Soltaninejad and Mitra Rahimi; Investigation, Writing - original draft, and writing - review \& editing: All authors.

\section{Conflict of interest}

The authors declared no conflict of interest.

\section{Acknowledgements}

We wish to thank the staff of Loghman Hakim Hospital Poison Center for their assistance in conducting this study.

\section{References}

[1] Bawaskar HS, Bawaskar PH. Scorpion sting: Update. Journal of the Association of Physicians of India. 2012; 60(1):46-55. [PMID]

[2] Dehghani R, Fathi B. Scorpion sting in Iran: A review. Toxicon. 2012; 60(5):919-33. [DOI:10.1016/j.toxicon.2012.06.002] [PMID]

[3] Al Abri S, Al Rumhi M, Al Mahruqi G, Shakir AS. Scorpion sting management at tertiary and secondary care emergency departments. Oman Medical Journal. 2019; 34(1):9-13 [DOI:10.5001/omj.2019.02] [PMID] [PMCID]

[4] Ebrahimi V, Hamdami E, Moemenbellah-Fard MD, Ezzatzadegan Jahromi S. Predictive determinants of scorpion stings in a tropical zone of south Iran: Use of mixed seasonal autoregressive moving average model. Journal of Venomous Animals and Toxins including Tropical Diseases. 2017; 23:39. [DOI:10.1186/ s40409-017-0129-4] [PMID] [PMCID]

[5] Khatony A, Abdi A, Fatahpour T, Towhidi F. The epidemiology of scorpion stings in tropical areas of Kermanshah province, Iran, during 2008 and 2009. Journal of Venomous Animals and Toxins including Tropical Diseases. 2015; 21:45. [DOI:10.1186/s40409015-0045-4] [PMID] [PMCID]

[6] Chippaux JP. Epidemiology of envenomations by terrestrial venomous animals in Brazil based on case reporting: From obvious facts to contingencies. Journal of Venomous Animals and Toxins including Tropical Diseases. 2015; 21:13. [DOI:10.1186/ s40409-015-0011-1] [PMID] [PMCID]

[7] Mahshidfar B, Basir Ghafouri H, Yasinzadeh MR, Mofidi M, Rezai M, Abbasi S. et al. Demographics of scorpion sting in Iran: A cross sectional study. Emergency (Tehran). 2017; 5(1):e77. [DOI:10.22037/emergency.v5i1.18276] [PMID] [PMCID]

[8] Sanaei-Zadeh H, Marashi SM, Dehghani R. Epidemiological and clinical characteristics of scorpionism in Shiraz (2012-2016) Development of a clinical severity grading for Iranian scorpion envenomation. Medical Journal of the Islamic Republic of Iran. 2017; 31:27. [DOI:10.18869/mjiri.31.27] [PMID] [PMCID]

[9] Nejati J, Saghafipour A, Rafinejad J, Mozaffari E, Keyhani A, Abolhasani A, et al. Scorpion composition and scorpionism in a high-risk area, the southwest of Iran. Electronic Physician. 2018; 10(7):7138-45. [DOI:10.19082/7138] [PMID] [PMCID]

[10] Moosavy SH, Shahi M, Rafinejad J, Zare S, Madani A, Navidpour S. Epidemiological aspect of scorpion sting in Bandar $\mathrm{Ab}$ bas, Iran, during 2009-2011. Electronic Physician. 2016; 8(4):228690. [DOI:10.19082/2286] [PMID] [PMCID]

[11] Jalali A, Pipelzadeh MH, Sayedian R, Rowan EG. A review of epidemiological, clinical and in vitro physiological studies of envenomation by the scorpion Hemiscorpius lepturus (Hemiscorpiidae) in Iran. Toxicon. 2010; 55(2-3):173-9. [DOI:10.1016/j. toxicon.2009.09.012] [PMID]

[12] Rafizadeh S, Rafinejad J, Rassi Y. Epidemiology of scorpionism in Iran during 2009. Journal of Arthropod-borne Diseases. 2013; 7(1):66-70. [PMID] [PMCID]

[13] Alkahlout BH, Abid MM, Kasim MM, Haneef SM. Epidemiological review of scorpion stings in Qatar. The need for regional management guidelines in emergency departments. Saudi Medical Journal. 2015; 36(7):851-5. [DOI:10.15537/smj.2015.7.11749] [PMID] [PMCID]

[14] Dehghani R, Kassiri H. Geographical distribution of scorpion Odontobuthus doriae in Isfahan Province, central Iran. Journal of Arthropod Borne Disease. 2017; 11(3):433-40. [PMID] [PMCID]

[15] Cesaretli Y, Ozkan O. Scorpion stings in Turkey: Epidemiological and clinical aspects between the years 1995 and 2004. The Revista do Instituto de Medicina Tropical de São Paulo. 2010; 52(4):215-20. [DOI:10.1590/S0036-46652010000400010] [PMID]

[16] Baradaran M, Jalali A, Naderi-Soorki M, Jokar M, Galehdari H. First transcriptome analysis of Iranian scorpion, Mesobuthus eupeus venom gland. Iranian Journal of Pharmceutical Research. 2018; 17(4):1488-502. [PMID] [PMCID]

[17] Reis MB, Zoccal KF, Gardinassi LG, Faccioli LH. Scorpion envenomation and inflammation: Beyond neurotoxic effects. Toxicon. 2019; 167:174-9. [DOI:10.1016/j.toxicon.2019.06.219] [PMID]

[18] Jalali A, Rahim F. Epidemiological review of scorpion envenomation in Iran. Iranian Journal of Pharmaceutical Research. 2014; 13(3):743-56. [PMID] [PMCID]

[19] Bahloul M, Turki O, Chaari A, Bouaziz M. Incidence, mechanisms and impact outcome of hyperglycaemia in se- 
vere scorpion-envenomed patients. Therapeutic Advances in Endocrinology and Metabolism. 2018; 9(7):199-208. [DOI:10.1177/2042018818772779] [PMID] [PMCID]

[20] Krishna Murthy KR. Treatment of scorpion envenoming syndrome: Need for scientific magnanimity. Journal of Indian Medical Association. 2013; 111(4):254-9. [PMID]

[21] Bouaziz M, Bahloul M, Kallel H, Samet M, Ksibi H, Dammak H. et al. Epidemiological, clinical characteristics and outcome of severe scorpion envenomation in South Tunisia: Multivariate analysis of 951 cases. Toxicon. 2008; 52(8):918-26. [DOI:10.1016/j.toxicon.2008.09.004] [PMID]

[22] Brown N, Landon J. Antivenom: The most cost-effective treatment in the world? Toxicon. 2010; 55(7):1405-7. [DOI:10.1016/j.toxicon.2010.02.012] [PMID]

[23] Valavi E. [Step by step treatment of scorpion sting in south west of Iran (Persian)]. Jundishapur Scientific Medical Journal. 2016; 15(1):117-24. https://www.magiran.com/ paper/1520922?lang=en

[24] Bosnak M, Ece A, Yolbas I, Bosnak V, Kaplan M, Gurkan F. Scorpion sting envenomation in children in southeast Turkey. Wilderness \& Environmental Medicine. 2009; 20(2):118-24. [DOI:10.1580/07-WEME-OR-098RR3.1] [PMID] 